\title{
Modelling the effect of screening on the spread of HIV infection in a population with variable inflow of infective immigrants
}

\author{
Issa Shabani ${ }^{1}$, Estomih S. Massawe ${ }^{1 *}$ and Oluwole Daniel Makinde ${ }^{2}$ \\ ${ }^{1}$ Mathematics Department, University of Dar es Salaam, P. O. Box 35062, Dar es Salaam, Tanzania. \\ ${ }^{2}$ Institute for Advance Research in Mathematical Modelling and Computations, Cape-Peninsula University of \\ Technology, P. O. Box 1906, Bellville 7535, South Africa.
}

Accepted 8 August, 2011

\begin{abstract}
This paper examines the combined effects of screening and variable inflow of infective immigrants on the spread of HIV/AIDS (human immunodeficiency virus/acquired immune deficiency syndrome) in a population of varying size. A nonlinear deterministic mathematical model for the problem is proposed and analysed qualitatively using the stability theory of differential equations. The results show that the reproductive number $R_{0}>1$ as the rate of inflow of infective immigrants increases leading to persistence of the disease in the population. However, the presence of screening greatly reduces the spread of HIV/AIDS. Numerical simulation of the model is implemented to investigate the sensitivity of certain key parameters on the spread of the disease.
\end{abstract}

Key words: Human immunodeficiency virus/acquired immune deficiency syndrome, screening, infective immigrants, reproductive number, stability analysis, numerical simulation.

\section{INTRODUCTION}

The acquired immunodeficiency syndrome (AIDS) emerged in 1981 and has become an important sexuality transmitted disease throughout the world. Moreover, the link between infectious diseases and population mobility must be understood in relation to the different forms, conditions and patterns of migration, which have very different influences on the distribution and spread of infectious diseases. For example in low-income countries, economic migration has played a crucial involvement in the evolution of the HIV/AIDS (human immunodeficiency virus/acquired immune deficiency syndrome) epidemic. However, research shows that internal and cross-border migrants, particularly male migrant workers are at greater risk of HIV infection and are more likely to spread the disease when they return home (Bozzette, 2005). Mathematical models have been used extensively in research into the epidemiology of HIV/AIDS to help improve our understanding of the major

\footnotetext{
${ }^{{ }}$Corresponding author. E-mail: emassawe@uccmail.co.tz.
}

contributing factors to the pandemic. From the initial models of Anderson et al. (1986), May and Anderson (1987) and Anderson (1988), various refinements have been added into modelling frameworks and specific issues have been addressed by researchers (Hethcote and Van Ark, 1992; Perelson and Nelson, 1999; McCluskey, 2003; Hsieh and Chen, 2004). Tripathi et al. (2007) presented a theoretical framework for transmission of HIV/AIDS with screening of unaware infectives. In all the aforementioned studies, the direct recruitment of infectives due to immigration has not been taken into account though it affects the dynamics of the disease to a significant level. It is well known that the immigrants can either be susceptible or infective and the infective immigrants play a crucial role on the spread of the disease. It is therefore essential to consider the direct inflow of infective immigrants to understand the dynamics of the spread of the disease. A very little attention has been paid to study the combined effects of screening and direct inflow of infective immigrants on the spread of HIV/AIDS in a community. Naresh et al. (2009) developed a model for the spread of HIV/AIDS in a 


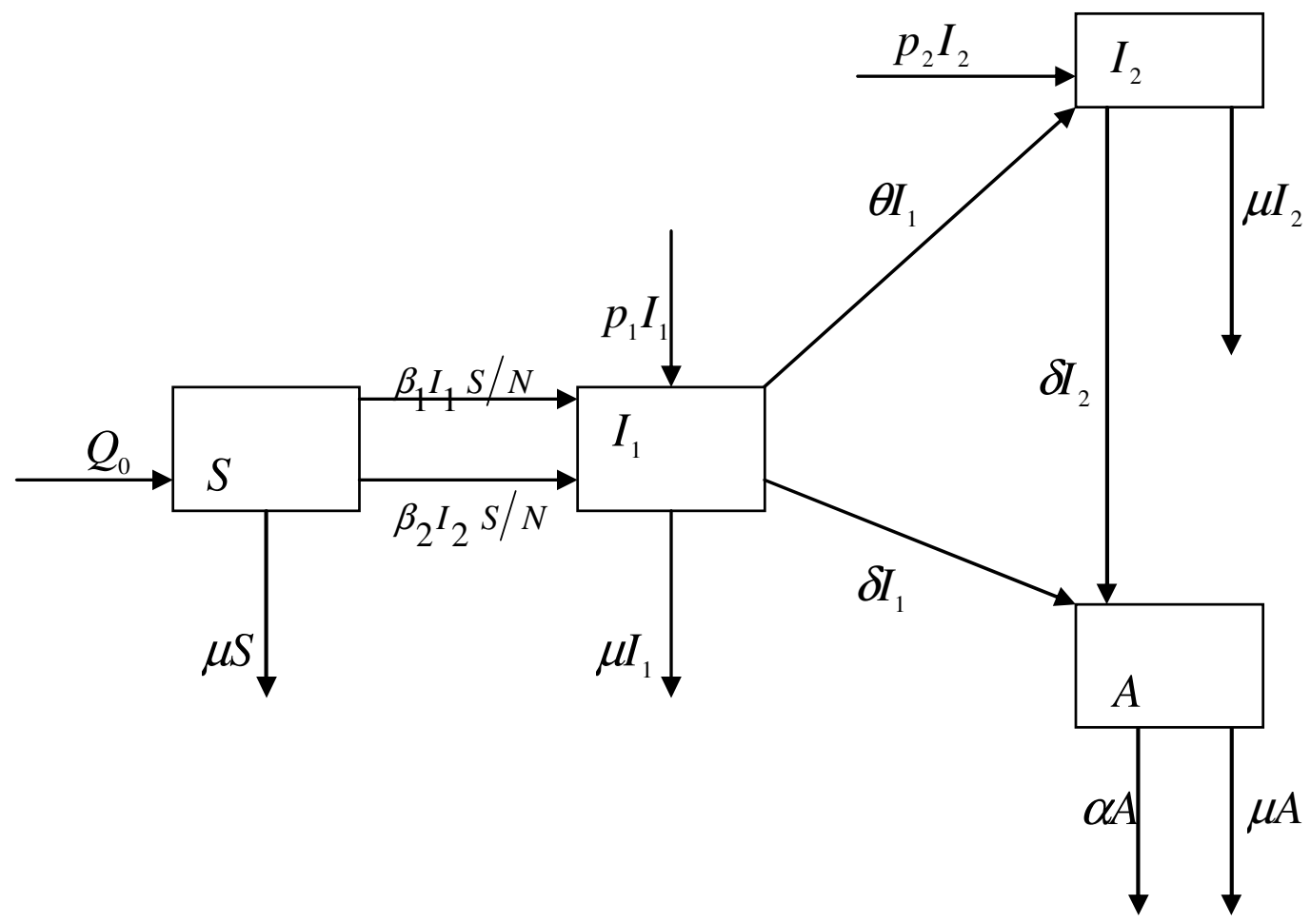

Figure 1. Flow diagram of the model.

community taking into account the constant inflow of infective immigrants. The objective of this present study is to investigate the combined effects of screening and direct inflow of infective immigrants on the spread of HIV/AIDS disease.

The model assumed that the rates of inflow of aware and unaware infective immigrants into the population vary. This essentially extends the earlier work of Tripathi et al. (2007) and Naresh et al. (2009) to include the combined impact of screening, variable inflow of aware and unaware infective immigrants on the HIV transmission dynamics. In the following study, the model is formulated, analysed and solved. Pertinent results are presented graphically and discussed qualitatively.

\section{MATHEMATICAL MODEL}

In modelling the disease dynamics, the population size $N(t)$ is divided into four subclasses of susceptibles $(S)$, unaware infectives $\left(I_{1}\right)$, aware infectives $\left(I_{2}\right)$ and that of AIDS patients $(A)$ with natural mortality rate $\mu$ in all the classes. The recruitment rate into the susceptible class is represented by $Q_{0}$ and the susceptibles become infected via sexual contacts with infectives class. The probabilities of disease transmission per contact by unaware and aware infectives are given as $\beta_{1}$ and $\beta_{2}$, respectively. It is reasonable to assume that $\beta_{2}<\beta_{1}$ because on becoming aware of the infection after screening, the infectives may choose to use preventive measures and change behaviour while $\theta$ is the detection rate for the unaware infectives. Let $0 \leq P_{1} \leq 1$ and $0 \leq P_{2} \leq 1$ represent the inflow rate of unaware and aware infective immigrants, respectively. Here $\delta$ is the rate of movement from infectious class to AIDS class and the AIDS related death rate is $\alpha$. The total population at any given time $t$ is given by:

$$
N(t)=S(t)+I_{1}(t)+I_{2}(t)++A(t)
$$

Taking into account of the aforementioned consideration, the transfer diagram of the model is shown in Figure 1. From the flow diagram, the dynamics of the disease is governed by the following system of nonlinear ordinary differential equations:

$$
\left\{\begin{array}{l}
\frac{d S}{d t}=Q_{0}-S\left(\frac{\beta_{1} I_{1}+\beta_{2} I_{2}}{N}\right)-\mu S, \\
\frac{d I_{1}}{d t}=p_{1} I_{1}+S\left(\frac{\beta_{1} I_{1}+\beta_{2} I_{2}}{N}\right)-(\theta+\delta+\mu) I_{1}, \\
\frac{d I_{2}}{d t}=p_{2} I_{2}+\theta_{1}-(\delta+\mu) I_{2}, \\
\frac{d A}{d t}=\delta_{1}+\delta_{2}-(\alpha+\mu) A .
\end{array}\right.
$$


With nonnegative initial conditions. Replacing $S=N-I_{1}-I_{2}$ $-A$ in the model of Equation 2, we obtain:

$$
\begin{aligned}
& \frac{d N}{d t}=Q_{0}+p_{1} I_{1}+p_{2} I_{2}-\mu N-\alpha A \\
& \frac{d I_{1}}{d t}=p_{1} I_{1}+\left(N-I_{1}-I_{2}-A\right)\left(\frac{\beta I_{1}+I_{2}}{N}\right)-(\theta+\delta+\mu) I_{1}, \\
& \frac{d I_{2}}{d t}=p_{2} I_{2}+\theta_{1}-(\delta+\mu) I_{2}, \\
& \frac{d A}{d t}=\delta_{1}+d_{2}-(\alpha+\mu) A,
\end{aligned}
$$

With $N(0) \geq 0, I_{1}(0) \geq 0, I_{2}(0) \geq 0, A(0) \geq 0$

Continuity of right-hand side of the Equation 3 and its derivative imply that the model is well posed for $N>0$. It is assumed that all dependent variables and parameters of the model are non-negative.

\section{Theorem}

Let $N(0) \geq 0, I_{1}(0) \geq 0, I_{2}(0) \geq 0, A(0) \geq 0, \quad$ then the solution of $\left\{S, I_{1}, I_{2}, A\right\}$ of the system (3) are positive for all $\mathrm{t} \geq 0$.

\section{Proof}

From the system (3), we obtain the inequality expression:

$$
\frac{d N}{d t} \leq Q_{0}-\mu N
$$

Which gives:

$$
N(t) \leq \frac{Q_{0}}{\mu}-C e^{-\mu t}
$$

As $t \rightarrow \infty$, we obtain $0 \leq N(t) \leq Q_{d} / \mu$. Hence all feasible solution of system (3) enter region:

$$
\left\{\left(S, I_{1}, I_{2}, A\right) \in R_{+}^{4}: 0 \leq N \leq \frac{Q_{0}}{\mu}\right\},
$$

Similar proof can be established for positivity of $S, I_{1}, I_{2}, A$.

\section{MODEL ANALYSIS}

The nonlinear system in Equation 2 will be qualitatively analyzed so as to find the conditions for existence and stability of a disease free equilibrium point (Gomes et al., 2004). Analysis of the model allows us to determine the impact of screening and inflow of infective immigrants on the spread of the diseases. Also on finding the reproductive number $R_{0}$, one can determine if the disease become endemic in a population or not.

\section{Disease free equilibrium (DFE)}

The disease free equilibrium of the model in Equation 2 is obtained by setting:

$$
\frac{d S}{d t}=\frac{d I_{1}}{d t}=\frac{d I_{2}}{d t}=\frac{d A}{d t}=0 .
$$

At disease free equilibrium, we have $I_{1}=I_{2}=A=0$ and Equation 2 becomes:

$$
Q_{0}-\mu S=0
$$

Therefore, the disease free equilibrium (DFE) denoted by $\vartheta_{0}$ of the model in Equation 2 is given by:

$$
\vartheta_{0}=(S, 0,0,0)=\left(\frac{Q_{0}}{\mu}, 0,0,0\right), \quad \mu>0
$$

\section{Local stability of DFE}

The disease free equilibrium of the model (3) was given by:

$$
\vartheta_{0}=(S, 0,0,0)=\left(\frac{Q}{\mu}, 0,0,0\right), \quad \mu>0
$$

The local stability of $\vartheta_{0}$ was established by using the next generation operator method on the system (3). The basic reproduction number $R_{0}$ is defined as the effective number of secondary infections caused by typical infected individual during his entire period of infectiousness (Diekmann et al., 1990). This definition is given for the models that represent the spreading of infection in a population. It is obtained by taking the largest (dominant) eigenvalue (spectral radius) of: 
$\left[\frac{\partial F_{i}\left(\vartheta_{0}\right)}{\partial x_{j}}\right] \cdot\left[\frac{\partial V_{i}\left(\vartheta_{0}\right)}{\partial x_{j}}\right]^{-1}$

Where:

$F_{i}$ is the rate of appearance of new infection in compartment $i$,

$V_{i}^{+}$is the transfer of individuals into compartment $i$,

$V_{i}^{-}$is the transfer of individuals out of the compartment $i$ by all other means,

$\vartheta_{0}$ is the disease-free equilibrium.

Consequently,

$\left(\begin{array}{c}f_{1} \\ f_{2}\end{array}\right)=\left(\begin{array}{c}\left(\beta_{1} I_{1}+\beta_{2} I_{2}\right) S \\ N \\ 0\end{array}\right)$.

By linearization approach, the associated matrix at disease-free equilibrium is given by:

$F=\left(\begin{array}{ll}\frac{\partial f_{1}}{\partial I_{1}}\left(\vartheta_{0}\right) & \frac{\partial f_{1}}{\partial I_{1}}\left(\vartheta_{0}\right) \\ \frac{\partial f_{2}}{\partial I_{2}}\left(\vartheta_{0}\right) & \frac{\partial f_{2}}{\partial I_{2}}\left(\vartheta_{0}\right)\end{array}\right)=\left(\begin{array}{cc}\beta_{1} & \beta_{2} \\ 0 & 0\end{array}\right)$,

and

$\left(\begin{array}{c}v_{1} \\ v_{2}\end{array}\right)=\left(\begin{array}{c}(\theta+\delta+\mu) I_{1}-p_{1} I_{1} \\ (\delta+\mu) I_{2}-p_{2} I_{2}-\theta I_{1}\end{array}\right)$.

Again by linearization we get:

$V=\left(\begin{array}{ll}\frac{\partial v_{1}}{\partial I_{1}}\left(\vartheta_{0}\right) & \frac{\partial v_{1}}{\partial I_{2}}\left(\vartheta_{0}\right) \\ \frac{\partial v_{2}}{\partial I_{1}}\left(\vartheta_{0}\right) & \frac{\partial v_{2}}{\partial I_{2}}\left(\vartheta_{0}\right)\end{array}\right)=\left(\begin{array}{cc}\theta+\delta+\mu-p_{1} & 0 \\ -\theta & \delta+\mu-p_{2}\end{array}\right)$,

And,

$V^{-1}=\left(\begin{array}{cc}\frac{1}{\left(\theta+\delta+\mu-p_{1}\right)} & 0 \\ \frac{\theta}{\left(\theta+\delta+\mu-p_{1}\right)\left(\delta+\mu-p_{2}\right)} & \frac{1}{\left(\delta+\mu-p_{2}\right)}\end{array}\right)$
Therefore:

$F V^{-1}=\left(\begin{array}{cc}\frac{\beta\left(\delta+\mu-p_{2}\right)+\beta \theta}{\left(\theta+\delta+\mu-p_{1}\right)\left(\delta+\mu-p_{2}\right)} & \frac{\beta\left(\theta+\delta-\mu-p_{1}\right)+\beta_{2} \theta}{\left(\delta+\mu-p_{2}\right)\left(\theta+\delta+\mu-p_{1}\right)} \\ 0 & 0\end{array}\right)$

The eigenvalues of $F V^{-1}$ are $\left(0, \frac{\beta_{1}\left(\delta+\mu-p_{2}\right)+\beta_{2} \theta}{\left(\theta+\delta+\mu-p_{1}\right)\left(\delta+\mu-p_{2}\right)}\right)$. It follows that the basic reproduction number for the model problem in Equation (3) denoted by $R_{0}$ is given as:

$R_{0}=\frac{\beta_{1}\left(\delta+\mu-p_{2}\right)+\beta_{2} \theta}{\left(\theta+\delta+\mu-p_{1}\right)\left(\delta+\mu-p_{2}\right)}$.

The disease free equilibrium is locally asymptotically stable if $R_{0}<1$ and unstable if $R_{0}>1$. In order to assess the contribution of $l_{1}$ and $l_{2}$ in terms of $\beta_{1}, p_{1}$ and $\beta_{2}, p_{2}$ from Equation 15, we let:

$$
\begin{aligned}
& R_{0 a}=\frac{\beta_{1}}{\left(\theta+\delta+\mu-p_{1}\right)}, \\
& R_{0 b}=\frac{\beta_{2} \theta}{\left(\theta+\delta+\mu-p_{1}\right)\left(\delta+\mu-p_{2}\right)}
\end{aligned}
$$

Then:

$R_{0}=R_{0 a}+R_{0 b}$

\section{Lemma}

The disease free equilibrium of the model (3) in the absence of infective immigrants is locally asymptotically stable if $R_{0}<1$ and unstable if $R_{0}>1$.

\section{REMARK}

It is clear from Equation 17 that $R_{0 a}>R_{0 b}$ which implies that unaware infectives $I_{1}$ have a significant contribution on the transmission of the infection and keeping the disease endemic in the population via $\beta_{1}$ and $p_{1}$ compared to the aware infectives $I_{2}$ via $\beta_{2}$ and $p_{2}$. In the absence of infection and infective immigrants, the population size approaches a steady state $Q_{0} / \mu$. However, as long as the infective immigrants are entering into the population, the disease free equilibrium will become unattainable making $R_{0}>1$. This is in agreement 
Table 1. Numerical values of sensitivity indices of $R_{0}$.

\begin{tabular}{cc}
\hline Parameter symbol & Sensitivity Index \\
\hline$\beta_{1}$ & +1.6499 \\
$\beta_{2}$ & +0.6499 \\
$\delta$ & -1.9333 \\
$\theta$ & -0.4215 \\
$\mu$ & -0.6905 \\
$p_{1}$ & +2.400 \\
$p_{2}$ & +0.100 \\
\hline
\end{tabular}

with the results of Naresh (2009) for constant inflow of infective immigrants. However, the present of screening may reduce the spread of the infection if it is given enough attention. Moreover, in the absence of infective immigrants (that is $P_{1}=0, P_{2}=0$ ), the results in Equations 15 to 16 reduces to that of Tripathi et al. (2007).

\section{Global stability of DFE}

The small influx of infective immigrants in the presence of screening may not generate large outbreaks if $R_{0}<1$. In order to establish global stability of DFE when $R_{0}<1$, we employed the comparison approach (Diekmann et al., 1990). The rate of change of the variables representing the infected components of the system (3) can be written as:

$\left(\begin{array}{c}\frac{d I_{1}(t)}{d t} \\ \frac{d I_{2}(t)}{d t}\end{array}\right)=(F-V)\left(\begin{array}{l}I_{1}(t) \\ I_{2}(t)\end{array}\right)-\left(\begin{array}{c}\beta_{1} I_{1}(t)+\beta_{2} I_{2}(t)-\left(\frac{\beta I_{1}+\beta_{2} I_{2}}{N}\right) S(t) \\ 0\end{array}\right)$

It follows that:

$$
\left(\begin{array}{l}
\frac{d I_{1}(t)}{d t} \\
\frac{d I_{2}(t)}{d t}
\end{array}\right) \leq(F-V)\left(\begin{array}{l}
I_{1}(t) \\
I_{2}(t)
\end{array}\right)
$$

Given that all the eigenvalues of the matrix $(F-V)$ have negative real parts, it follows that the inequality (19) is globally stable for $R_{0}<1$ and $\left(l_{1}, I_{2}\right) \rightarrow(0,0)$ as $t \rightarrow \infty$.

\section{Sensitivity analysis}

We perform sensitivity analysis in order to determine the relative importance of model parameters to disease transmission. In determining how best to reduce human mortality and morbidity due to HIV, it is necessary to know the relative importance of the different factors responsible for its transmission. We compute numerical sensitivity indices to enable us to single out parameters that have a high impact on $R_{0}$ and which should be targeted by intervention strategies. The normalized forward sensitivity index of a variable to a parameter is a ratio of the relative change in the variable to the relative change in the parameter. When a variable is a differentiable function of the parameter, the sensitivity index may be alternatively defined using partial derivatives.

$\mathrm{H}_{m}^{R_{0}}=\frac{m}{R_{0}} \frac{\partial R_{0}}{\partial m}$

For example, using the parameter values highlighted in the following study, the sensitivity index of $R_{0}$ with respect to $\beta_{1}$ is given as:

$$
\mathrm{H}_{\beta_{1}}^{R_{0}}=\frac{\beta_{1}}{R_{0}} \frac{\partial R_{0}}{\partial \beta_{1}}=1.6499
$$

Table 1 shows the sensitivity indices of other parameters with respect to $R_{0}$. From Table 1 , the most sensitive parameter augmenting the spread of HIV infection is the rate of inflow of unaware infective immigrants' $P_{1}$ into the population. This is followed by contact rate of unaware HIV infected individuals $\beta_{1}$ with susceptibles and contact rate of susceptibles with aware HIV infected individuals $\beta_{2}$ and the inflow rate of aware infective immigrants $P_{2}$. Moreover, it is noteworthy that screen rate of unaware HIV infectives $\theta$, natural mortality rate $\mu$ and transfer rate of aware and unaware HIV infectives $\delta$ to AIDS class contribute to a decline in the spread of HIV infection. 
Table 2. Parameter values used in simulations.

\begin{tabular}{ccl}
\hline Parameter symbol & Parameter value $\left(\boldsymbol{y r}^{-1}\right)$ & Source \\
\hline$\beta_{1}$ & +1.3440 & Trimpathi et al. (2007) \\
$\beta_{2}$ & +0.6000 & Estimated \\
$\delta$ & +0.5600 & Estimated \\
$\theta$ & +0.6000 & Estimated \\
$\mu$ & +0.2000 & Estimated \\
$\alpha$, & +0.1000 & Trimpathi et al. (2007) \\
$p_{1}$ & +0.8000 & Estimated \\
$p_{2}$ & +0.0800 & Trimpathi et al. (2007) \\
\hline
\end{tabular}

\section{Endemic equilibrium}

To obtain an endemic equilibrium $\omega=\left(N^{*}(t), I_{1}^{*}(t), I_{2}^{*}, A^{*}(t)\right)$, we set each equation in Equation 3 to zero and express each dependent variable in terms of $I_{1}^{*}$ at equilibrium point and we obtain:

$$
\begin{aligned}
& N^{*}=\frac{\left.q(\alpha+\mu)\left(\delta+\mu-p_{2}\right)+((\alpha+\mu) \theta-\delta \theta+\delta+\mu)-(\alpha+\mu)\left(\delta+\mu-p_{2}\right) p_{1}\right) I_{1}^{*}}{\mu(\alpha+\mu)\left(\delta+\mu-p_{2}\right)}, \\
& I_{1}^{*}=\frac{\left(\beta_{1}+\beta_{2} \tau+\gamma\right) r_{1}}{\left(\beta_{1}+\beta_{2} \tau\right)(1+\tau+\phi)+r_{2}\left(\beta_{1}+\beta_{2} \tau+\gamma\right)}, \\
& I_{2}^{*}=\frac{\theta I_{1}^{*}}{\left(\delta+\mu-p_{2}\right)}, \\
& A^{*}=\frac{\delta\left(\theta+\delta+\mu-p_{2}\right) I_{1}^{*}}{(\alpha+\mu)\left(\delta+\mu-p_{2}\right)},
\end{aligned}
$$

Where,

$$
\begin{array}{ll}
\tau & =\frac{\theta}{\left(\delta+\mu-p_{2}\right)}, \quad \gamma=\left(p_{1}-(\theta+\delta+\mu)\right), \\
\phi & =\frac{\delta\left(\delta+\theta+\mu-p_{2}\right)}{(\alpha+\mu)\left(\delta+\mu-p_{2}\right)}, \quad r_{1}=\frac{Q_{0}}{\mu}, \\
r_{2}=\frac{(\alpha+\mu)\left(\delta+\mu-p_{2}\right)+\left(\delta+\theta+\mu-p_{2}\right)-(\alpha+\mu) \theta}{\mu(\alpha+\mu)\left(\delta+\mu-p_{2}\right)} .
\end{array}
$$

We note that $N^{*}(t), I_{1}^{*}(t), I_{2}^{*}(t)$ and $A^{*}(t)$ exist and are positive if $R_{0}>1$.

\section{NUMERICAL SIMULATION}

To study the dynamical behaviour of the model numerically, the system in Equation 3 is integrated by fourth order Runge-Kutta method using the parameter values given in Table 2. The parameter values of $\beta_{2}, \delta$, $\theta, \mu$ and $p_{1}$ are estimated for simulation purposes only. In Figure 2, the distribution of population with time is shown for all classes. It is found that susceptible population decreases with time due to inflow of infective immigrants leading to an increase in the rate on infection. Initially, unaware infective class increases with time and then reaches its equilibrium position. Similar trend is observed with the population of aware infectives due to screening. Moreover, it is interesting to note that the AIDS population decreases due to screening of unaware infected population. This can be attributed to a change in risk behaviour and prevention. Figure 3 shows the variation in the population unaware infectives with an increase in the inflow rate of infective immigrants. As the rate of inflow of infective immigrants increase, unaware HIV infectives population increases as well. However, the infection is at its lowest level when infective immigrants are not allowed to enter into the population. This shows clearly that the inflow of infective immigrants contributes largely to the spread of the disease. Figure 4 shows that the AIDS population increases with an increase in the inflow rate of infective immigrants. In Figure 5 we observe that as the rate of screening increases, the population of unaware infectives decreases leading to an increase in the population of aware infectives as expected.

Consequently, a reduction in the spread of the disease will occur. Thus, to keep the spread of the HIV/AIDS epidemic under control, the screening of unaware 


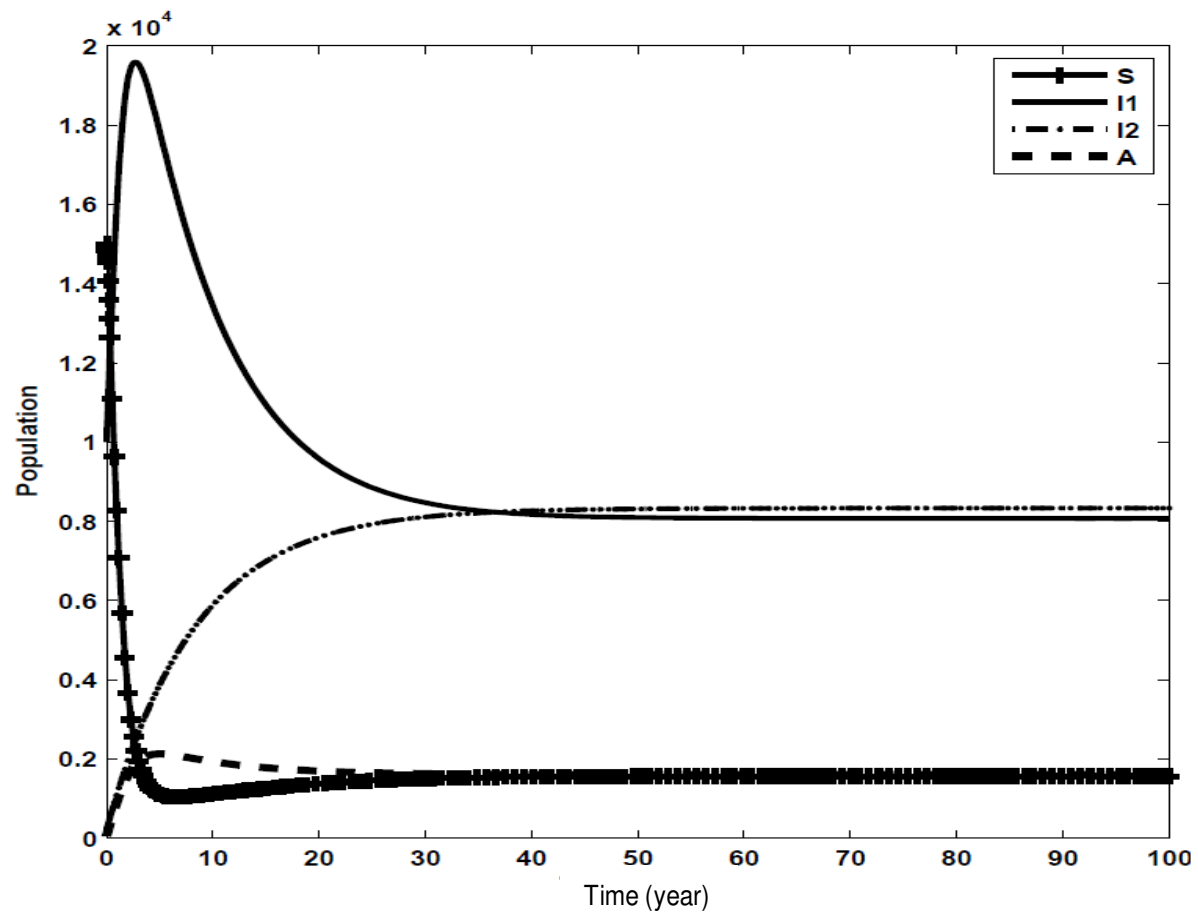

Figure 2. Variation of population in different classes for the given parameter values.

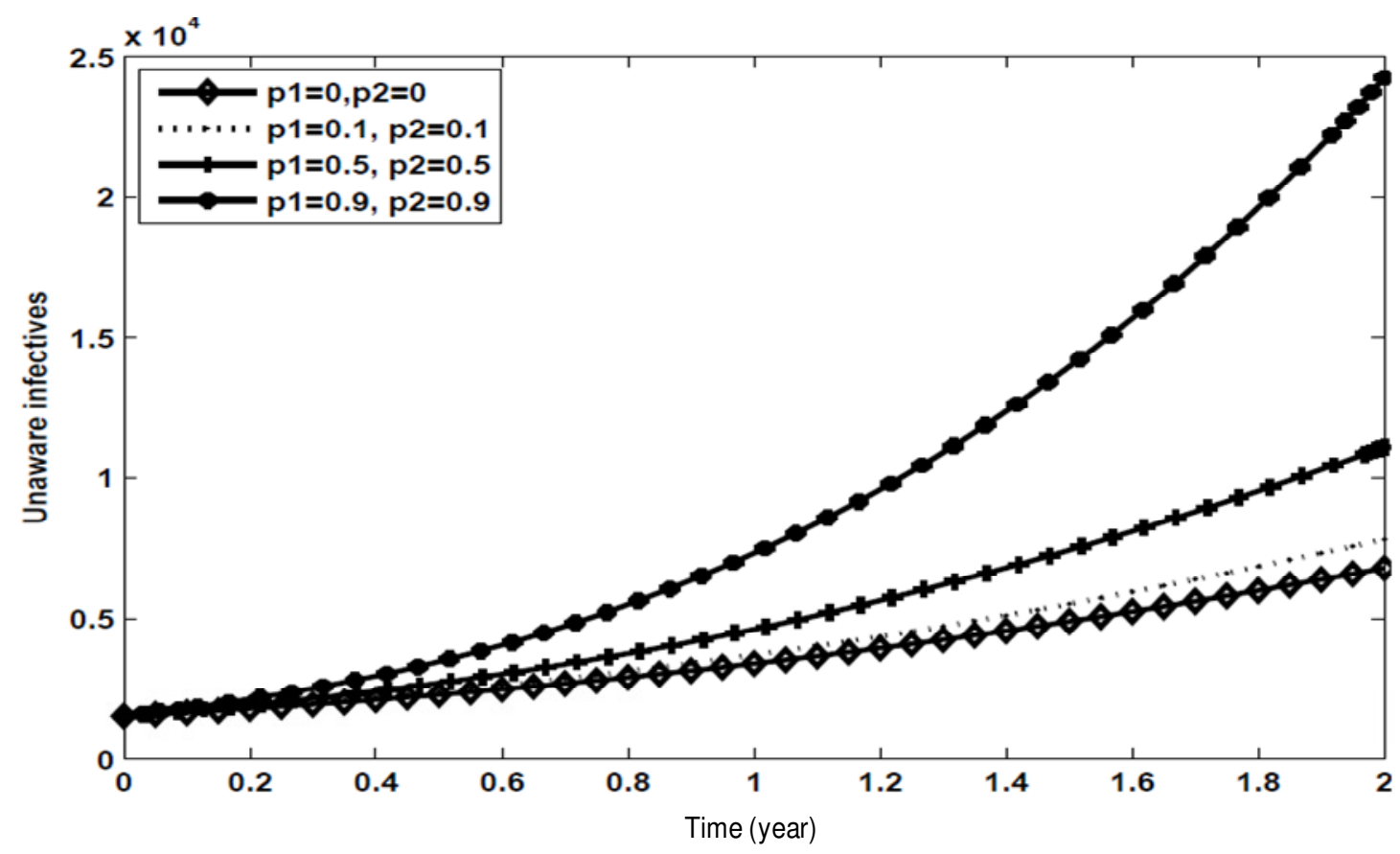

Figure 3. Effect of inflow of infective immigrants on unaware HIV infected class.

infectives both within the population and as well as immigrants must be intensified, coupled behaviour changes so that they can either abstain from sexual interaction or use preventive measures.

\section{Conclusions}

In this paper, a non-linear model for the combined effects of screening and variable inflow of infective immigrants 


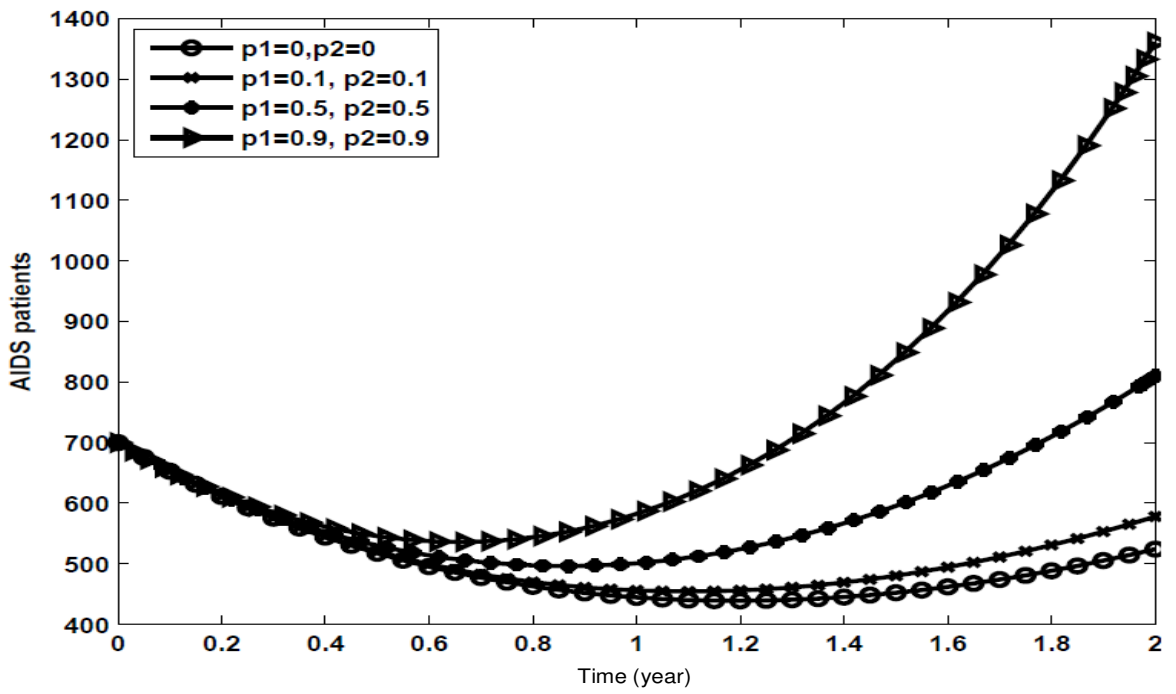

Figure 4. Effect of inflow of infective immigrants on AIDS population.
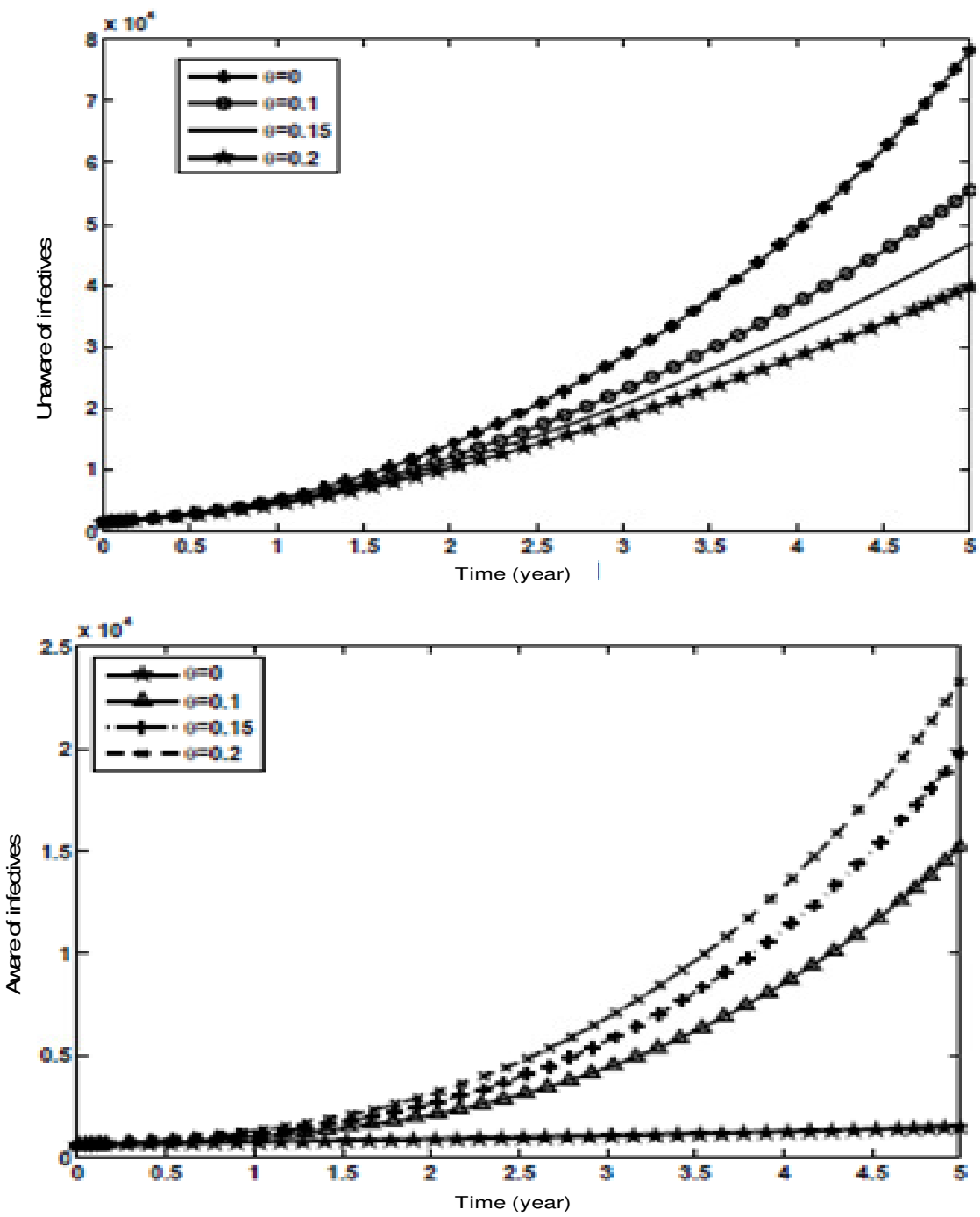

Figure 5. Effect of screening on unaware and aware infectives. 
on the spread of HIV/AIDS is investigated. It was shown that there exists a feasible region where the model is well posed and for which a unique disease free equilibrium point exists in the absence of infective immigrants. The sensitivity of the key parameters on the spread of HIV/AIDS revealed that an increase in the screening rate coupled with the rate of progression from infectives to AIDS class may lead to a decline in the spread of HIV/AIDS.

\section{ACKNOWLEDGEMENTS}

The authors wish to thank the anonymous referees for their useful comments.

\section{REFERENCES}

Anderson RM (1988). The role of mathematical models in the study of HIV transmission and the epidemiology of AIDS, J. AIDS, 1: 241-256.

Anderson RM, Medly GF, May RM, Johnson AM (1986). A preliminary study of the transmission dynamics of the Human Immunodeficiency Virus (HIV), the causative agent of AIDS, IMA J. Math. Appl. Med. Biol., 3: 229-263.
Bozzette SA (2005). Routine Screening for HIV Infection-Timely and Cost-effective, New Engl. J. Med., 352:620-621.

Diekmann O, Heesterbeek JAP, Metz JAJ (1990). On the definition and the computation of the basic reproduction ratio $R_{0}$ in models for infectious diseases in heterogeneous populations, J. Math. Biol., 28: 365-382.

Hethcote HW, Van Ark JW (1992). Modelling HIV Transmission and AIDS in the United States, in: Lect. Notes Biomath., Vol. 95, Springer, Berlin.

Hsieh YH, Chen $\mathrm{CH}$ (2004). Modelling the social dynamics of a sex industry: Its implications for spread of HIV/AIDS, Bull. Math. Biol., 66: 143-166.

May R. M., Anderson R. M., (1987). Transmission dynamics of HIV infection, Nature, 326: 137-142.

McCluskey CC (2003). A model of HIV/AIDS with staged progression and amelioration, Math. Biosci., 181: 1-16.

Naresh R, Tripathi A, Sharma D (2009). Modelling and analysis of the spread of AIDS epidemic with immigration of HIV infectives. Math. Comput. Mod. 49: 880-892.

Perelson AS, Nelson PW (1999). Mathematical analysis of HIV-1 dynamics in vivo, SIAM Rev., 41 (1): 3-44.

Tripathi A, Naresh R, Sharma D (2007). Modelling the screening of unaware infectives on the spread of HIV infection, Appl. Math Comput., 184: 1053-1068. 